\title{
Kommentare/Gespräch
}

\section{Zum Selbstverständnis gewerkschaftlich organisierter Richter und Staatsanwälte}

Unter Richtern und Staatsanwälten, die der Gewerkschaft ötv angehören und die dort die »Fachgruppe Richter und Staatsanwälte « bilden, wird in jüngerer Zeit verstärkt die Diskussion um das Selbstverständnis ${ }^{t}$ geführt. Es geht dabei sowohl um das Selbstverständnis innerhalb der Justiz als auch um das Selbstverständnis als Gewerkschafter. Es liegt auf der Hand, daß sich eine besonders brisante Diskussion ergibt, wenn man beides aufeinander bezieht und nach dem Selbstverständis gewerkschaftlich organisierter Juristen mit Blick auf beide Institutionen fragt. Ich werde einige Gründe für die verstärkte Diskussion um das Selbstverständnis benennen und zugleich die Notwendigkeit einer solchen Diskussion zu begründen versuchen (Teil 1.). Danach will ich versuchen, aus meiner Sicht das Selbstverständnis der gewerkschaftlich organisierten Kollegen zu skizzieren (Teil 2.). ${ }^{2}$

I. Notwendigkeit und Ursachen der neuentfachten Diskussion um das Selbstverständnis

\section{I.I. Justiz}

Die Altersstruktur der Justiz hat sich schlagartig verändert. Die Justizstatistik von $1979^{3}$ weist aus, daß von den knapp 19000 Richtern und Staatsanwälten in der

\footnotetext{
I Die Diskussion wird nicht systematisch und organisiert geführt; vielfach verbirgt sie sich hinter Debatten und in Beiträgen zu justiz- und rechtspolitischen Tagesthemen einerseits und zur Gewerkschaftspolitik (etwa zur Diskussion um den Entwurf eines neuen DGB-Grundsatzprogramms) andererseits. Die Diskussion entwickelı sich "wildwüchsig«, aber vielleicht gibt gerade das ihr den Reiz und die Innovationskraft. An einigen Stellen ist die Diskussion schon öffentlich geworden. Genannt seien: Hans-Egon Bender (Richter am Bundessozialgericht), Zwischen Staatsgewalt und Arbeiterbewegung, in: ötv-Magazin 7/78, S. I 8 f.; Ulrich Vultejus, Die Linke und die Dritte Gewalt, in: Die Linke, Bilanz und Perspektiven für die 8oer, hrsg. v. Hermann L. Gremliza und Heinrich Hannover, Hamburg 1980, S. 220 ff.; ötv Weser-Ems, Justiztag '79, 2 r. - 22. 9. 1979, Dokumentation (mit Beträgen von Rothländer, Däubler, Hans-Egon Bender und Karl-Heinz Werner), Bremen 1980. Die Zeitschrift »ötv in der Rechtspflege " (Mitteilungen der Fachgruppen in den Bezirken Niedersachsen, Weser-Ems, Hamburg, c/o ötv Bezirk Niedersachsen, Dreyerstr. 6, 3000 Hannover r; bis jetzt sind 2s Hefte erschienen) zeigt seit Jahren eine mehr praktische Seite der Selbstverständnisdiskussion, indem dort der Justizalltag als Anlaß zur Reflexion genommen wird. Die vielleicht interessantere Diskussion ist erst im Begriff, eine öffentliche Dimension zu gewinnen: In verschiedenen Bezirken (vgl. etwa für Bremen, die AG »Entwicklung des Rechtsstaats und berufliche Praxis", s. KJ r/8 I, S. 18 ff.) oder in fachspezifischen Arbeitskreisen (bisher vor allem in der Arbeitsgerichtsbarkeit) wird über Funktion und Stellenwert einer ihre Abhängigkeit begreifenden und um ihre Unabhängigkeit kämpfenden Justiz im demokratischen und sozialen Rechtsstaat und damit über das Selbstverständnis der in der Justiz tätigen Individuen wie der Institution explizit diskutiert.

2 Das Selbstverständnis, das ich skizziere, ist in erster Linie dasjenige der Richter. Für die Staatsanwälte müssen vor allem die Aussagen modifiziert werden, die ihre Grundlage in dem spezifischen Richterstatus mit der grundgesetzlichen Garantie der persönlichen und sachlichen Unabhängigkeit haben. Gelegentlich ist ausdrücklich darauf hingewiesen, daß eine Aussage für beide Berufsgruppen gilt.

3 Nach DRiZ 1979, S. 288 f., 320 u. 350 f.
} 
Bundesrepublik jeweils ca. 950 bzw. I 000 den Jahrgängen 1943 und 1944 angehörten. Dem entspricht, daß nicht mehr nur wenige ältere Richter und Staatsanwälte (insbesondere Richter der Arbeits- und Sozialgerichtsbarkeit) der Gewerkschaft angehören. Vielmehr sind unter den jüngeren Kollegen, soweit sie Neigung hatten, in den Justizdienst zu gehen und man sie hineingelassen hat, eine große Zahl derjenigen, die aus der "68er-Generation « stammen, die in den Jahren seit $1972 / 73$ verstärkt aus den ehemaligen Referendarverbänden heraus sich schon während der Ausbildung der Gewerkschaft ötv anschlossen; vereinzelt sind schon Absolventen der einphasigen Ausbildungsgänge anzutreffen. Für sie alle gilt, daß ihnen die sozialwissenschaftliche Fundierung des Studiums nicht nur ein Mittel zur Effektivierung der Ausbildung bedeutet; sie sind vielmehr eher zu erkennen geneigt, daß der Richter nicht als einsames Individuum oder Mitglied eines kollegialen Organs Recht spricht, sondern sehr wohl Produkt seines gesellschaftlichen Umfeldes ist und wiederum auf dieses einwirkt.

Es läßt sich von daher ein zahlenmäßiges Wachsen der gewerkschaftlichen Präsenz in der Justiz feststellen. Die Prozentzahlen der Gewerkschafter unter den Richtern und Staatsanwälten, bezogen auf die Gesamtzahl, liegen im Schnitt der Bundesrepublik zwischen 5 und $10 \%$. Dabei ist überall festzustellen, daß die Gewerkschafter in den Gerichten der Eingangsinstanz bzw. im Eingangsamt am stärksten vertreten sind. Die Verteilung über die einzelnen Gerichtsbarkeiten ist (mit Ausnahme der Finanzgerichtsbarkeit, die im übrigen auch das Eingangsamt $R_{2}$ hat) breit gestreut. Richter in höheren Ämtern, vor allem solche mit Behördenleiterfunktion, sind auch vertreten, aber eher unterdurchschnittlich, bezogen auf den Stellenkegel. In einzelnen Bundesländern halten sich heute Hochburgen in der Arbeits- und Sozialgerichtsbarkeit (so insbesondere in Niedersachsen, Hessen und Nordrhein-Westfalen). Es wird nicht überraschen und ist doch ebenso bezeichnend wie beängstigend, daß in CDUregierten Ländern (mit Ausnahme des altliberalen Baden-Württemberg und Niedersachsens) Richtergewerkschafter schwach oder nahezu überhaupt nicht zu finden sind, während sie ihre starke Position in sozialliberal bzw. sozialdemokratisch regierten Ländern haben. Die Spitze bildet dabei Bremen, wo jetzt knapp $25 \%$ der Richter und Staatsanwälte Gewerkschaftsmitglied sind. ${ }^{4}$

Was folgt daraus? Die Justiz ist, anders als es bisher die Arbeiten zur Herkunft und Sozialisation der Juristen auswiesen, kein monolithischer Block mit einheitlichen Wertvorstellungen und entsprechendem esprit de corps mehr ${ }^{42}$. Ansatzweise spiegelt sich das plurale Bild der Gesellschaft der Bundesrepublik, wie es etwa seinen Niederschlag im Ausgang von Wahlen findet, auch in der Justiz wieder. Ich sage wohlgemerkt »ansatzweise «: In der Justiz ist die »Mitte « noch immer konservativer als die politische "Mitte« der Bundesrepublik Deutschland. Das bedeutet nicht unbedingt, daß die Mehrzahl der Richter der politischen Rechten, also dem konservativen Flügel der FDP, der CDU/CSU oder weiter rechts stehenden Gruppen zuzurechnen ist; eine große Zahl der hier gemeinten Richter würde sich dagegen verwahren.

Vermittelt über Ausbildung und berufliche Sozialisation wird aber konservatives Denken auch anderen eigen. Dies zeigt sich in der (mitgliedschaftlichen) Identifikation mit dem Deutschen Richterbund, dessen programmatische Aussagen sowie Initiativen und Stellungnahmen zu (rechts)politischen Ereignissen und Gesetzge-

\footnotetext{
4 Vgl. zur Mitgliederentwicklung dort KJ 1/81, S. 18. Bei $25 \%$ liegt interessanterweise auch der Stimmenanteil, den bei neueren Richterratswahlen Kandidaten der ötv-Fachgruppe auch in Flächenstaaten und dort erzielen, wo die Mitgliederzahl weit darunter liegt.

4a Auch der gestiegene Anteil der Frauen trägt dazu bei: Es gab 1979 I 2,23\% Richterinnen und ca. 10\% Staatsanwältinnen (ebenfalls noch DRiZ 1979, S. 350 f.).
} 
bungsvorhaben regelmäßig mit denjenigen der CDU/CSU übereinstimmen, wenn nicht sie rechts überholen. Immerhin läßt sich aber feststellen, daß der Deutsche Richterbund, in dem Mitglied zu sein für Richter und Staatsanwälte bis in die 6oer Jahre eine quasi-automatische Folge des Eintritts in den Beruf war, in seiner Mitgliederzahl stagniert. Eine gegenüber gewerkschaftlichen Positionen indifferente bis interessierte Gruppe unter den Richtern und Staatsanwälten, deren persönliche Zusammensetzung breit gestreut ist, sieht bewußt vom Beitritt in den Richterbund ab, vollzieht aber andererseits nicht den Schritt zum Gewerkschaftsbeitritt. In einzelnen Orten, Regionen oder Gerichtsbarkeiten wird diese Gruppe allein oder zusammen mit den Gewerkschaftern bereits zur »stillen Mehrheit«.

\section{I.2. Gewerkschaft}

Richter und St tatsanwälte gehören von der Profession her Institutionen an, die historisch zumeist »auf der anderen Seite « gestanden haben als die Organisationen der Arbeiterbewegung. Uberdies sind sie von vornherein im Beruf funktional Vorgesetzte anderer Bediensteter, die schon eher und breiter gewerkschaftlich organisiert waren.' Daraus resultierten in der Anfangszeit der Fachgruppe und vereinzelt noch heute (berechtigte) Reserven innerhalb der Gewerkschaften selbst gegenüber denjenigen Angehörigen dieser Berufsgruppen, die Mitglied in der Gewerkschaft geworden sind.

Inzwischen sind die Vorbehalte weitestgehend abgebaut. Die Fachgruppe kann sich auf allen Ebenen der gewerkschaftlichen Hierarchie Gehör verschaffen und sich, wenn die Organisation sich die Vorstellungen zu eigen macht, über die ötv nach außen artikulieren; umgekehrt macht sich die Gewerkschaft den Sachverstand der Fachgruppe zu Nutzen, wenn es darum geht, rechtspolitische Positionen zu formulieren und Forderungen anzumelden.

\subsection{Probleme rechtspolitischer Arbeit in der Gewerkschaft}

Bisher bestand die Tätigkeit der Fachgruppe auf Kreis-, Bezirks- und Bundesebene im wesentlichen darin, einerseits ad hoc aktuelle justizpolitische Ereignisse und Themen aufzugreifen, andererseits im Rahmen der Gesetzgebungstätigkeit in Bund und Ländern Stellungnahmen abzugeben; daneben fiel die Arbeit in Richter- und Personalräten an. Probleme konnten bei der gewerkschaftlichen Willensbildung auftreten: etwa wenn es darum ging, eine (inhalt lich vielleicht auch noch brisante) zur Außendarstellung und insbesondere zur Abgrenzung gegenüber dem Richterbund notwendige Stellungnahme zu aktuellen justizpolitischen Ereignissen abzugeben und dabei nicht mit den satzungsmäßigen Gegebenheiten der Organisation zu kollidieren. Zugleich stellte sich mehr und mehr heraus, daß es für eine kleine Gruppe von aktiven Kollegen in Sprecherfunktionen unmöglich ist und in blanken Dezisionismus ausartet, wenn man punktuell und ohne vorherigen Prozeß grundsätzlichen kollektiven Nachdenkens zu einzelnen justizpolitischen Fragen Positionen bezieht: vom Kontaktsperregesetz bis zur Reform der Richterpersonalvertretung, von der Trennscheibe zwischen Verteidiger und Mandant bis zur Staatshaftung, von der einheitlichen Neuregelung des Verwaltungsprozesses bis zur computergerechten Ausgestaltung der Formulare für den Mahnbescheid, von der Reform der Juristenausbildung bis zu derjenigen des $\$ 218$.

Natürlich wird die Fachgruppe sich nicht verweigern, wenn die rechtspolitische Situation es erfordert bzw. die Gewerkschaft oder der DGB es von ihr erwartet, einen Standpunkt zu dieser oder jener Frage nach außen zu artikulieren. Es setzt sich aber 
die Erkenntnis durch, daß zur Fundierung und letzten Endes auch zur Verstärkung der justizinternen wie gewerkschaftsinternen, vor allem aber öffentlichen Durchsetzungskraft eine breite Diskussion über das Selbstverständnis der Richter und Staatsanwälte im demokratischen und sozialen Rechtsstaat und unter den konkreten politischen und gesellschaftlichen Verhältnissen der Bundesrepublik Deutschland ebenso unerläßlich ist wie das Nachdenken über die Funktion der Justiz insgesamt unter diesen Rahmenbedingungen.

\section{Selbstverständnis}

\subsection{Der Richter als Arbeitnehmer}

Die Gewerkschafter unter den Richtern (ebenso wie die Staatsanwälte) begreifen sich als Arbeitnehmer. Sie leben von den Einnahmen, die sie durch Hingabe ihrer Arbeitskraft an einen Dritten (hier: die Länder bzw. den Bund) erzielen. Insoweit sind sie abhängig Beschäftigte (Arbeitnehmer) wie andere Angehörige des öffentlichen Dienstes auch. Daß sie, was ihre richterliche Entscheidungstätigkeit und ihren besonderen Status angeht, unabhängig und nur dem Gesetz unterworfen sind, daß sie, soweit sie »hauptamtlich und planmäßig endgültig angestellt « sind, grundsätzlich nicht abgesetzt oder versetzt werden können (Art. $97 \mathrm{GG}$ ), ändert daran nichts. Freilich müssen sie sich bewußt sein, daß sie eine Gruppe sind, die nur Angehörige des höheren Dienstes umfaßt und deren Mitglieder mit überkommenen Privilegien ausgestattet sind (»freie« Arbeitszeit der Richter). ${ }^{6}$

Begreifen sich die Richter als Arbeitnehmer, so ist der Beitritt zur Gewerkschaft konsequent, anders als wenn man sich als Stand begreift und sich demgemäß in einer Standesorganisation wie dem Richterbund organisiert (mag dieser sich auch modisch nach außen gelegentlich fast wie eine Gewerkschaft geben). Aus dem Selbstverständnis als Arbeitnehmer leiten sich aber auch ganz konkrete Positionen für die Interessenvertretung und politische Argumentation ab: Ein Richter, der sich als Arbeitnehmer versteht, wird die Forderung nach einem einheitlichen Recht der Beschäftigten des öffentlichen Dienstes (freilich unter Beachtung einiger verfassungsrechtlicher Vorgaben für die Richter) unterstützen; er wird sich - ein Schritt in diese Richtung - für einen Abbau der disziplinarrechtlichen Fesseln für Beamte und Richter und gegen eine grundrechtsbeschränkende Praxis des jetzt geltenden Rechts einsetzen. Er wird sich für eine Ausweitung der Rechte der Richterräte in Richtung einer echten Mitbestimmung und für die Abschaffung der unzureichend demokratisch legitimierten Präsidialräte einsetzen; die Mitarbeit in den bestehenden Richterräten und die Zusammenarbeit mit den Personalräten der übrigen Bediensteten leitet sich ebenso daraus ab. Der Richter, der sich als Arbeitnehmer versteht, wird seine arbeitsplatzbezogene Interessenvertretung (was etwa die Stellenvermehrung und die Ausstattung der Gerichte angeht) mit der der übrigen Bediensteten abstimmen; er leistet so zugleich einen Beitrag zum Abbau des in den Gerichten noch weit mehr als in anderen Behörden verbreiteten Standes- und Laufbahngruppendenkens. Die Zusammenarbeit mit anderen Gewerkschaftskollegen im Rahmen von Betriebsgruppen ist eine weitere Konsequenz. Gegen die - auch für gewerkschaftlich organisierte Richter und Staatsanwälte bestehende - Gefahr des Korporatismus gibt selbst dies alles zwar keine Garantie; es besteht aber immerhin eine gewisse Wahrscheinlichkeit, daß sie erkannt und ihr gegengesteuert wird.

Auch die Frage, an welchen Punkten eine Abgrenzung zum Deutschen Richterbund

6 Bender (Fn. ५), ebda. 
geboten ist und wo auf der anderen Seite Ansatzpunkte für eine Zusammenarbeit liegen, läßt sich von dem Ausgangspunkt "Der Richter als Arbeitnehmer" klarer beantworten.

2.2. Der unabhängige Richter als Garant individueller und kollektiver Freibeiten Der Richter ist, wie gezeigt, abhängig Beschäftigter des Bundes oder eines der Länder. Er ist damit aber nicht »Staatsdiener «, oder jedenfalls nur in einem sehr beschränkten bzw. modifizierten Sinne: Der Staat ist sein »Arbeitgeber «- dies knüpft direkt an den Gedanken vom Richter als Arbeitnehmer an -, er stellt den Richter an und alimentiert ihn; seinen besonderen Dienst erweist der Richter der Gesellschaft und dem Staat aber darin, daß er in seiner aus der übrigen Staatsgewalt herausgenommenen Stellung nach dem Gewaltenteilungsprinzip die individuellen Freiheiten der Bürger (und der Assoziationen von Bürgern), auch und besonders gegen den Staat, schützt. Er ist nicht "funktionierender « Teil einer einheitlich organisierten Staatsgewalt, sondern ist (wie die Justiz als Ganzes) ein Organ, das der idealtypischen Konstruktion nach geradezu dafür geschaffen ist, aus der im übrigen einheitlich organisierten Staatsgewalt »herauszufallen «, dieser gegenzusteuern und sich ihr dort, wo es das Gesetz gebieter, zugunsten des Bürgers zu versagen bzw! den Schutz durch die staatlichen Machtmittel dem Bürger zugute kommen zu lassen. Nun wäre es gefährlich, der Ideologie der richterlichen Unabhängigkeit aufzusitzen und die idealtypische Konstruktion mit der Realität zu verwechseln. Ein erster, bescheidener Schritt zur Erkämpfung und Durchsetzung der richterlichen Unabhängigkeit wird daher sein, sich die eigene soziale, rechtspolitische und wissenschaftliche Abhängigkeit bewußt zu machen. Nur von diesem Ausgangspunkt her wird es möglich sein, im demokratischen und sozialen Rechtsstaat die bürgerlich-liberale Doktrin vom unabhängigen Richter und der unabhängigen »Dritten Gewalt« zu aktualisieren und zu materialisieren.

\section{Folgerungen}

Die erste Folgerung bezieht sich auf die juristische Arbeitsweise, im besonderen auf die Methode der Gesetzesanwendung und -interpretation'. Hier wird eine Arbeitsweise anzustreben sein, die sich mit der Formel »nicht unkritischer Gesetzespositivismus« umschreiben läßt.

Das Votum für den Gesetzespositivismus bedeutet vor allem ein Votum für den demokratischen Verfassungs- und Gesetzgeber und ein Votum für die strikte Einhaltung formellen Rechts. Es knüpft an die demokratische Tradition deutscher Rechtslehre und Justiz (Thoma, Anschütz, Kelsen, Heller, Fraenkel, Kirchheimer, F. Neumann u. a.; vgl. auch unten 2.4.) in der Weimarer Republik an.

Mit dem bloßen Beharren auf der Position des Gesetzespositivismus kann es aber nicht sein Bewenden haben. Der Versuch, die theoretisch begründete strikte Bindung des Richters an das demokratische Gesetz als allein handlungsleitende Formel zur Lösung jederzeit auftauchender praktischer Problemlagen zu verwenden, schlägt fehl. Dazu ist das geschriebene Gesetz oft zu unklar, ausfüllungsbedürftig bzw. lückenhaft. Auch wird sich in ganzen Rechtsgebieten (insbesondere im Arbeits- und Sozialrecht, wenn der Gesetzgeber die Normierung unterläßt und die Regelung dem gesellschaftlichen Antagonismus überläßt) die Notwendigkeit für die Gerichte

\footnotetext{
7 Ich gebe im folgenden den Diskussionsstand der AG der Bremer Fachgruppe "Entwicklung des Rechtsstaats und berufliche Praxis (s. Fn. I) wieder, wie er insbesondere in zwei unveröffentlichten Arbeitspapieren vom Okt. 1980 von Viggo Eiberle-Herm und Heinrich Schnitger festgehalten ist.
} 
ergeben, rechtsschöpferisch bzw. -ergänzend tätig zu werden. Schließlich ist vielfach durch (ihrerseits mit Hilfe interpretations- bzw. ausfüllungsbedürftiger Normen zu praktizierende) verfassungskonforme Interpretation einfaches Recht zu konkretisieren.

Die Formel vom "nicht unkritischen Gesetzespositivismus« versucht deshalb einerseits, durch die Hervorhebung der strikten Gesetzesbindung einer Auflösung der Legalordnung zu wehren, und andererseits, dem unvollkommenen Charakter einzelner Normen, Gesetzesteile, Gesetze oder Rechtsgebiete in Orientierung an der Verfassung gerecht zu werden, beides häufig in Auseinandersetzung mit vorkonstitutionellen, in Universität, Referendariat und Justizpraxis tradierten und reproduzierten "herrschenden Meinungen « und juristischen Kunstlehren.

Die Formel ist thesenhaft, pauschal und vorläufig. Es wird notwendig sein, sie in Arbeitsgruppen aus den einzelnen Gerichtsbarkeiten und Fachgebieten anhand von Problemen aus der Alltagspraxis auf die Probe zu stellen, zu verfeinern und fortzuschreiben, sie gegebenenfalls auch ganz oder teilweise zu verwerfen. Dabei wird zu prüfen sein, ob diesem Modell des Verhältnisses von Justiz und parlamentarischem Gesetz nicht eine idealisierte Demokratievorstellung entspricht (Parlamentarismuskritik, Demokratietheorie!) und ob dabei nicht die handlungsleitende Funktion des Gesetzes für die Justiz überschätzt wird. Anders gesagt: Ob nicht der Zersetzungsprozeß der traditionellen Legalordnung, der mit einem Funktions- und Machtzuwachs der Justiz einhergeht und seinen Ausdruck in Rechtsfiguren wie "Wertordnung« sowie zunehmenden Güter- und Interessenabwägungen der entscheidenden Instanz findet, unvermeidbar (weil den besonderen Anforderungen des politischen und gesellschaftlichen Systems angemessen) ist und ob nicht die hier befürwortete Strategie der Rekonstruktion der Legalordnung einen Kampf gegen Windmühlenflügel bedeutet.

Der Fachgruppe kommt die Funktion der Organisierung der Diskussion dieser Probleme als ein wesentlicher inhaltlicher Bestandteil gewerkschaftlicher Justizpolitik zu. Das Verhältnis von Berufspraxis, Gewerkschaftsarbeit, Rechtspolitik und persönlichem Engagement in der beruflichen Praxis wird sich sodann näher bestimmen lassen.

Die Fortführung der Diskussion zur Arbeitsweise des »nicht unkritischen Gesetzespositivismus « würde überdies die juristische Methodendiskussion bereichern. Es ist ein grundsätzlicher Unterschied, ob jemand außerhalb der Justiz deren Funktionsweise analysiert oder ob ein Mitglied der Justiz bzw. eine Gruppe von Justizangehörigen bei der Analyse zugleich die höchst praktische Frage der sich ergebenden Praxisauswirkungen und der anzustrebenden Strategie notwendigerweise mitzubedenken hat. Die Diskussion wäre durch die Gleichzeitigkeit der drei Komponenten

- Praxisnähe

- empirische Forschung

- subtile Theoriebildung

gekennzeichnet; dies sind nicht von ungefähr die Eigenschaften, die den kritischen und höchst praktischen Rechts- und Justizsoziologen der Weimarer Republik zugeschrieben werden können. ${ }^{8}$

Eine zweite Folgerung betrifft den Verhandlungsstil und den Umgang mit der Offentlichkeit. Bei dem direkten Umgang mit den Betroffenen bzw. Parteien wird ein nicht-autoritärer Verhandlungsstil zu fordern und zu praktizieren sein. Die Offent-

8 So, bezogen auf Franz Neumann, Alfons Söliner in seinem Nachwort zu: Franz Neumann, Die Herrschaft des Gesetzes, Frankfurt/M. 1980, S. 365 . 
lichkeit ist nicht nur als ein papierener Grundsatz des Gerichtsverfassungsgesetzes, sondern als Verpflichtung der Justiz anzusehen, sich nicht zu verschanzen, sondern sich auf den Dialog mit der Gesellschaft einzulassen. Dies heißt insbesondere, daß die Immunisierung gegen Justizkritik abzubauen ist.

Die dritte Folgerung, die hier nur kurz benannt sein soll, betrifft das Verhältnis zu den Anwälten: Die Parteilichkeit der Anwälte ist zu respektieren. Es widerspricht fundamental der freien Advokatur, den Anwalt »zu einem Staatshörigen hinaufzudegradieren . $^{9}$

Schließlich ergeben sich im Inneren der Justiz eine ganze Reihe von Folgerungen für das Verhalten unter den gegenwärtigen gerichtsverfassungsrechtlichen und justizorganisatorischen Strukturen. Sie lassen sich so zusammenfassen, daß der Richter sich nicht weiter "verwalten" lassen darf, sondern daß er die Verwaltung der Justiz als seine Angelegenheit begreift. Die dem Modell nach einer demokratischen Selbstverwaltung der Gerichte, jedenfalls zum wichtigen Punkt der Geschäftsverteilung, entsprechende Konstruktion des Präsidiums ist derart mit Inhalt zu füllen, daß der Leiter des Gerichts wirklich nur noch für die vereinigten Richter die Geschäfte führt. Diejenigen Verwaltungsaufgaben, die sich unmittelbar und direkt auf die Rechtsprechung beziehen (wie etwa die Tätigkeit von Pressestellen) sind von der Gesamtheit der Richter und nicht vom Präsidenten als Justizverwaltungsaufgabe wahrzunehmen. Uberhaupt sind die Befugnisse der Justizverwaltung gegenüber den Richtern auf das strikt dienstrechtlich gebotene Maß herabzusetzen. Die entwürdigende und der Sache nach unsinnige Konstruktion der "Erprobung « erfahrener Richter im "Dritten Staatsexamen « bei dem jeweiligen Obergericht, insbesondere als informelle Voraussetzung für eine einigermaßen aussichtsreiche Bewerbung in ein anderes ("höheres «) Richteramt, ist abzuschaffen. Die Richter müssen sich gegen alle Einschüchterungsversuche durch die Justizhierarchie wehren, die in den verschiedensten Formen betrieben werden: durch selbstherrliche Entscheidungen der Obergerichte, die juristische Diskussion mit Schulmeisterei verwechseln; durch Beurteilungen, die das Weltbild des Beurteilenden zum Maß der Dinge machen; durch »kollegiale Gespräche«, die nicht wie unter Gleichen geführt werden.

Die Gefahr eines Abbaus der Kompetenzen der Eingangsgerichte, insbesondere des Amtsgerichts, ist zu beobachten und ihr entgegenzusteuern. Die Gefahr ergibt sich aus der zunehmenden Tendenz der Obergerichte, "Tabellen «, "Kataloge« und andere Richtlinien zwischen das Gesetz und den zur Entscheidung im Einzelfall berufenen Richter zu stellen; dies mag zwar auf der einen Seite der Gleichbehandlung dienen, führt aber auf der anderen Seite dazu, daß aus dem Richter ein Beamter in Sachbearbeiterfunktion mit entsprechender Mentalität wird; die nächste Stufe ist dann die Einrichtung computergerechter Verfahren...

Mit dem letzten Punkt ist, mehr als mit den vorhergehenden Folgerungen, der Bereich der Gesetzgebung berührt. Denn der beklagte Abbau der Kompetenzen der Eingangsgerichte ist auch gefördert worden durch die - unkritisch und unter technokratischen Gesichtspunkten bejubelte - Einführung des direkten Instanzenzuges von den Amtsgerichten zu den Oberlandesgerichten, etwa in Ordnungswidrigkeiten- und Familiensachen. Bezeichnenderweise hat dort gerade das Gericht über die Entscheidungen des Amtsrichters zu entscheiden, dessen Präsident oberster richterlicher Dienstvorgesetzter ist und bei dem das »Dritte Staatsexamen« abzuleisten ist...

9 Hans Dahs sen., Stellung und Grundaufgaben des Verteidigers, NJW r959, S. Irs8ff; vgl. zur Problematik des Verteidigers als Organ der Rechtspflege auch Ingo Müller, Rechtsstaat und Strafverfahren, Frankfurt/M. 1980, S. $208 \mathrm{ff}$. 
Aus den Folgerungen ergäben sich Forderungen an den Gesetzgeber. Ich habe mich jedoch auf die Folgerungen unter dem gegenwärtigen Rechtszustand beschränkt. Viele der de lege lata aufgestellten Forderungen sind bereits in den 6oer Jahren erörtert worden, z. T. ist der jetzige Gesetzeszustand, etwa hinsichtlich der Präsidialverfassung, der Richterwahl in verschiedenen Bundesländern und der Richterbesoldung, Produkt jener Diskussionen und Forderungen. ${ }^{\circ}$

\subsection{Die Gewerkschaft als Organisation der Justizbasis}

Wie sich aus dem Stellenkegel der Justiz ablesen läßt, sind die Mehrzahl der Richter

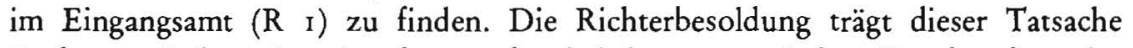
Rechnung, indem sie, wie schon vorher bei der automatischen Durchstufung der Richter im Eingangsamt von der Besoldungsgruppe A I 3 nach A I5, auch ohne formelle "Beförderung" den Richtern finanziell einen den Beamten zumindest entsprechenden Status gibt. Der Kunstgriff der Justizverwaltungen und der Gerichtspräsidenten hat in der Vergangenheit darin bestanden und besteht bis heute darin, über ein subtiles System von Verlockungen (Aussichten auf in der Hierarchie höher angesiedelte Ämter) und Einschüchterung (weniger durch die plumpen Mittel des Disziplinarverfahrens als vielmehr durch Nuancen in der Benotung) die Richter dazu zu bringen, sich in Konkurrenz untereinander in den Startlöchern für die (den meisten schon nach der statistischen Wahrscheinlichkeit unerreichbaren) höher dotierten Justizämter zu zerstreiten. Damit wird die Chance verspielt, das einheitliche, in allen Ämtern mit der Garantie der Unabhängigkeit ausgestattete Richteramt dazu zu nutzen, individuell und kollektiv über die Funktion der eigenen Tätigkeit und die Funktion der Justiz nachzudenken und die gewonnenen Erkenntnisse zugunsten der Klienten der Justiz einzusetzen. Bezeichnenderweise sind die Vorstände des Deutschen Richterbundes auf Bundes- und Landesebene durch eine Identität mit der höheren Ebene der Justizhierarchie gekennzeichnet.

Dem ist ein Selbstverständnis entgegenzusetzen, das die Gewerkschaft als Organisation der Justizbasis ansieht, wie es der geschilderten Zusammensetzung der Fachgruppe entspricht. Die Interessen derjenigen, die sich ihre richterliche Unabhängigkeit ständig auch durch Beharrlichkeit gegenüber ihren eigenen Verwaltungen erkämpfen müssen, sind nicht durch Angehörige dieser Verwaltung und durch Akzeptieren der Sozialisationsmuster ihrer Repräsentanten zu vertreten.

\subsection{Die Fachgruppe in der Tradition des "Republikanischen Richterbundes}

Es gibt für die gewerkschaftlich organisierten Justizjuristen ein historisches Vorbild: den "Republikanischen Richterbund « in der Weimarer Republik. Wie die Mitglieder dieser Vereinigung setzen sie sich für die konsequente Verwirklichung des demokratischen und sozialen Rechtsstaates auch im Bereich der Justiz und durch die Justiz ein.

Zwei Gesichtspunkte führen jedoch über die Tradition des Republikanischen Richterbundes hinaus. Der erste ist schon genannt: Die Fachgruppe versteht sich als Organisation der Justizbasis. Dies ist, unter den gegenüber der Weimarer Republik veränderten gesellschaftlichen Verhältnissen der Bundesrepublik, eine Konsequenz auch aus der Tatsache, daß es dem Republikanischen Richterbund nie gelungen ist, sich wirklich in der Justiz zu verankern, insbesondere auch nur eine starke Minderheitsposition unter den Amtsrichtern und Richtern der Landgerichte zu

Io Zur (damals auch, wenn nicht überwiegend, im Reformflügel des Deutschen Richterbundes geführten) Diskussion der 6oer Jahre vgl. die Beiträge in: Rudolf Wassermann (Hrsg.), Justizreform, Neuwied und Berlin 1970; zu den fortgeltenden Maximalforderungen vgl. Ulrich Vultejus, a. a. O. (Fn. 1), S. 232. 
erringen; der Republikanische Richterbund war vielmehr eine Organisation von Anwälten, Hochschullehrern, Gewerkschaftsjuristen, Beamten und daneben nur einigen Richtern und Staatsanwälten, die dann eher (republikanisch gesonnene) Behördenleiter denn Kollegen von der Justizbasis waren.

Der zweite Gesichtspunkt besteht in dem Schritt von der unabhängigen, aber notwendigerweise kleinen und elitären Organisation zum Beitritt in die Gewerkschaft des öffentlichen Dienstes, eine Konsequenz aus dem oben skizzierten Selbstverständnis als Arbeitnehmer.

Die Anknüpfung an die Tradition des Republikanischen Richterbundes bedeutet, auf dessen - freilich auf die konkrete historische Situation zwischen 1918 und 1933 bezogene - Arbeiten zur Gesetzgebung zur praktischen Rechts- und Justizpolitik, zur Justizkritik und zur politischen Soziologie der Justiz zurückzugreifen.

\subsection{Die Fachgruppe in der Reibe der gewerkschaftlichen Richterorganisationen Westeuropas}

Es existieren auch in anderen Ländern Westeuropas gewerkschaftliche Organisationen von Justizjuristen. Mit ihnen, von denen hier die französische Organisation "syndicat de la magistrature " und die italienische "magistratura democratica " beispielhaft genannt sein sollen, lassen sich eine Fülle von Gemeinsamkeiten feststellen, die eine verstärkte Kommunikation und Zusammenarbeit nahelegen.

Auf den ersten Blick scheint mehr für das Gegenteil zu sprechen, nämlich dafür, daß die nationalen Besonderheiten einem Vergleich oder gar einer Kooperation entgegenstehen: Die französischen und italienischen Organisationen leben jeweils in einer anderen politischen Kultur; sie profitieren im Zentralstaat Frankreich wie im regionalisierten Italien von einer ganz anderen politischen Offentlichkeit, insbesondere in der Form der Diskussion rechts- und justizpolitischer Ereignisse und Reflexion der Rolle der Justiz in den großen Blättern der jeweiligen Metropolen; in beiden Ländern finden die Klassenauseinandersetzungen offener statt und lassen die Fragwürdigkeit der Funktion der Justiz offenkundig werden; schließlich haben die dortigen Richtergewerkschaften eine andere, auch noch untereinander wieder verschiedene Organisationsform: »magistratura democratica « ist die linke Fraktion des einheitlichen italienischen Richterverbandes. Die französische Organisation "syndicat de la magistrature « ist eine kleine autonome Gewerkschaft der Richter und Staatsanwälte. Sie stellt dabei in Frankreich eine Besonderheit insoweit dar, als sie die sonst in Richtungsgewerkschaften gespaltenen Flügel der französischen politischen Linken in einer Fachgewerkschaft organisiert.

Trotz dieser Divergenzen drängt sich ein verstärkter Kontakt - er besteht bisher sporadisch und weniger auf institutioneller als auf persönlicher Ebene - bis hin zur Koordination und Kooperation auf. Denn allen Organisationen liegen ähnliche Ubberlegungen zum Selbstverständnis, insbesondere zu den zentralen Fragen des Verhältnisses zum demokratischen Gesetzgeber, zur Verteidigung der Legalordnung, zur Garantie der individuellen und kollektiven Freiheiten und zur Rolle des unabhängigen Richters einschließlich des Kampfes gegen die Bevormundung und Gängelung durch Justizhierarchie und -bürokratie zugrunde. Die französischen und

I Zum "syndicat de la magistrature * vgl. Udo Reifner, Gewerkschaftliche Orientierung von Anwälten in Frankreich, KJ 3/76, S. 258 ff; Hartmut Bäumer, Untersuchungsrichter Charette und der Unternehmer Chapron, KJ 3/76, S. 278 ff; Lothar Zechlin, Das Syndicat de la Magistrature, DuR I/79, S. $38 \mathrm{ff}$; zur "magistratura democratica" vgl. Christoph Ulrich Schminck, Zur Zerstörung der Rechtsorthodoxie in Italien, KJ I/73, S. 20 ff; ders., Magistratura Democratica (Bericht über einen sozialistischen Richterkongreß in Italien), KJ 4/75, S. $4^{\circ} 4 \mathrm{ff}$; ders., Beobachtungen zur Justizkrise in Italien, DuR $3 / 76, \mathrm{~S}$. $226 \mathrm{ff}$. 
italienischen Kollegen sind allenfalls in ihren Reflexionen und in ihrer gewerkschaftlichen und beruflichen Praxis schon weiter gediehen. Das mag auch dadurch gefördert sein, daß sie nicht, wie die Fachgruppe in der Bundesrepublik, ihre Aktionen und Uberlegungen jeweils auf die verschiedene Gesetzeslage und das jeweilige Ministerium in elf Bundesländern und zusätzlich im Bund (etwa zur Frage der Richtermitbestimmung!) zuschneiden (und zersplittern) müssen; vielmehr sehen sie sich jeweils nur einem Widerpart, nämlich dem nationalen Justizminister, gegenüber und finden eine einheitliche Gesetzeslage vor. Gemeinsam ist den beiden Organisationen "syndicat de la magistrature « und "magistratura democratica «, daß sie versuchen, zu einer Synthese von theoretischer Reflexion, beruflicher Praxis und gewerkschaftlicher Justizpolitik zu gelangen.

Aus Italien ließen sich z. B. Erfahrungen mit der Reaktion der Justiz auf lokale politische Kämpfe, insbesondere den »Häuserkampf, « übertragen; von Kollegen der »magistratura democratica « könnten auch berufspraktische Erfahrungen aufgegriffen werden, die sie bei der Reform der psychiatrischen Versorgung der Bevölkerung in Zusammenarbeit mit Ärzten der "Demokratischen Psychiatrie " erworben haben ${ }^{12}$. Aus Frankreich ließen sich z. B. Erfahrungen der Jugendrichter (die dort u. a. auch Funktionen unserer Vormundschaftsrichter und der Heimaufsicht wahrnehmen) über die Zusammenarbeit mit Sozialarbeitern und Trägern der Jugendhilfe, über ihren Versuch der Neudefinition der Rolle des Richters und die Grenzen, an die sie justizintern und außerhalb stoßen, verwerten ${ }^{13}$. Aus Frankreich wären auch besonders krasse Beispiele von Disziplinarfällen in der Justiz zu diskutieren, die zeigen, wie nervös und überzogen (oder planvoll und weitsichtig?) die Justiz auf die Tatsache reagiert, daß zu ihr jetzt auch eine nonkonforme starke Minderheit gehört, die auch noch für sich in Anspruch nimmt, das "Im Namen des Volkes « ${ }^{14}$ besonders ernst zu nehmen.

Es bleibt schließlich ein Grund, der die verstärkte Kontaktaufnahme, Koordination und Kooperation unter den westeuropäischen Richtergewerkschaften unabweisbar erscheinen läßt: Auf dem Gebiet der Repression findet auf Regierungsebene schon jetzt eine Koordination und Kooperation statt, die ihren Ursprung und ihre Rechtfertigung in der Verfolgung der ursprünglich politisch motivierten Gewaltkriminalität hat. ${ }^{15}$ Es ist im Moment zu befürchten, daß der »Europäische Rechtsraum « (L'espace européen de droit) sich auf ein gemeinsames Konzept und eine einheitliche Praxis der Beschränkung von Freiheitsrechten reduziert. Dagegen ist es notwendig, daß, entsprechend ihrem gemeinsamen Selbstverständnis, insbesondere die gewerkschaftlich organisierten Richter (und Staatsanwälte) in allen betroffenen Ländern gemeinsam und koordiniert in ihrer Berufspraxis den freiheitlichen Aspekt des geltenden Rechts wahren und über ihre Organisationen rechtspolitische Initiativen ergreifen bzw. unterstützen, die eine weitere übermäßige Einschränkung von Freiheitsrechten verhindern und umgekehrt den Schutz des Bürgers vor der Allmacht des Staates stärken.

Hans-Ernst Böttcher

12 Vgl. den Hinweis im Nachruf von Erich Wulff, Zum Tod von Franco Basaglia, in: Das Argument 124 (I980), S. 862 ff (864).

13 S. Heft 8o/8 r der Zeitschrift des »syndicat de la magistrature«, »justice«: Spécial Justice des Mineurs, Paris 1980.

14 Der Titel eines der Selbstzeugnisse des »syndicat de la magistrature ist eine Anspielung hierauf: Syndicat de la magistrature (Hrsg.), Au nom du peuple français (= Im Namen des (frz.) Volkes), Paris 1974.

1s Das französische Gesetz *sécurité/liberté « (veröffentlicht im "Journal officiel« vom 3. Februar 1981; vgl. die Hefte $77 / 78$ und 79 von "justice*, beide Paris 1980 , und die verschiedenen Beiträge in "Le Monde" vom 4. und 5 . Februar 1981) beschert den Franzosen einen Gesetzeszustand, der sich nur unwesentlich von dem unterscheidet, der spätestens seit dem "Razziengesetz« (Gesetz zur Änderung der Strafprozeßordnung vom I4. 4. 1978; BGBl. I, S. 497) in der Bundesrepublik gilt. 
blätter des informationszentrums dritte welt

Themen der letzten Nummern waren: + Tansania: Bürokratie behindert sozialistische Entwicklung + Dritte Welt im Spiegel der Massenmedien + Unterdrückung der Indios in Südamerika + Malaysia: Kampf um Wohnraum + Medizin in der Dritten Welt + Probleme landwirtschaftlicher Entwicklung + Indien: Ursachen und Formen des Elends der indischen Bauernbevölkerung + Argentinien: Unterdrückung und Widerstand + Energieversorgung der Dritten Welt + Chile: Faschismus im 5. Jahr + Mexiko: Elend der Bauern + Welthandelskonferenz V: Entwicklung für wen? + Kolumbien: Geschichte, Arbeiterbewegung, wirtschaftliche Lage +

Die in 8 Ausgaben pro Jahr erscheinende Zeitschrift + berichtet über die Lage der Dritten Welt, die Ursachen ihrer „Unterentwicklung" und über den Widerstand der unterdrückten Völker und Nationen

+ gibt Aufschluß über den Zusammenhang zwischen der kapitalistischen Wirtschaftsstruktur hier und dem Elend der Massen in der Dritten Welt

+ beleuchtet die "Entwicklungshilfe", ihre Hintergründe und Auswirkungen + setzt sich mit den Vorstellungen der bürgerlichen Entwicklungsländertheorie und -politik auseinander

+ berichtet über den Befreiungskampf der Völker in der Dritten Welt und den Aufbau unabhängiger, fortschrittlicher Staaten

+ will zu praktischer Solidarität mit dem Kampf der Dritten Welt um Freiheit und Unabhängigkeit anregen + gibt. Hinweise und Erfahrungsberichte aus der Solidaritätsarbeit.

Jahresabonnement bei 8 Nummern mit 50 - 60 Seiten: DM 35,- (Für Studenten, Zivildienstleistende und andere einkommensschwache Gruppen nach Selbsteinschätzung: DM 25,-).

- Informationszentrum Dritte Welt, Postfach 5328, D-7800 FREIBURG, Telefon: $0761 / 74003$

- Kostenloses Probeexemplar anfordern!

Bitte senden Sie mir ein Probeexemplar!

Name

Vorname

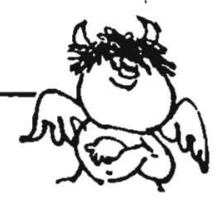

Strasse

PLZMohnort 